\title{
CARACTERIZACIÓN DE FRUTOS Y SEMILLAS DE ALGUNAS CUCURBITÁCEAS EN EL NORTE DEL PERÚ
}

\author{
CHARACTERIZATION OF FRUITS AND SEEDS OF SOME CUCURBITS \\ IN NORTHERN PERÚ
}

\author{
Guillermo E. Delgado-Paredes*, Consuelo Rojas-Idrogo, Ángela Sencie-Tarazona y Leopoldo Vásquez-Núñez
}

Facultad de Ciencias Biológicas, Universidad Nacional Pedro Ruiz Gallo. Ciudad Universitaria, Juan XXIII núm. 391. Lambayeque, Perú.

${ }^{\star}$ Autor para correspondencia: (guidelg2001@yahoo.es)

\section{RESUMEN}

Se colectó germoplasma de cucurbitáceas en el norte del Perú, en las regiones de Tumbes, Piura, Lambayeque y Cajamarca, para un total de 8 géneros, 14 especies y 202 accesiones, sobre todo de la región Lambayeque que tuvo el mayor número de accesiones. Este material genético se conserva desde 1988 hasta la actualidad a $10{ }^{\circ} \mathrm{C}$ de temperatura, con una tasa de viabilidad de las semillas de $90 \%$. Se evaluaron las características morfológicas de frutos y semillas de las especies Cucurbita ficifolia, C. moschata, C. maxima y Cucurbita sp., una variedad local conocida como "loche", y Lagenaria siceraria. Las accesiones de Cucurbita fueron muy variables en forma, tamaño y color de fruto, y forma y tamaño de semilla. Las accesiones de Lagenaria destacaron por la amplia variabilidad en forma y tamaño del fruto, en las que se reconocieron los tipos "calabazos", "lapas", "mates" y "checos", con numerosas formas intermedias. Se discute la posibilidad de que los genotipos "chuyán" y "loche", clasificados tradicionalmente como C. moschata, pudieran constituir subespecies. Se concluye que la costa norte del Perú es un centro de alta diversidad morfológica de C. moschata y L. siceraria, y la región altoandina de Cajamarca y Piura de C. ficifolia.

Palabras clave: Cucurbita ficifolia, C. moschata, Lagenaria siceraria, descriptores morfológicos, diversidad morfológica.

\section{SUMMARY}

Cucurbit germplasm was collected in northern Perú. Sampling covered the Tumbes, Piura and Cajamarca regions and gathered 8 genera, 14 species and 202 accessions. The highest number of accesions came from Lambayeque. Genetic material has been conserved since 1988 to this date at $10^{\circ} \mathrm{C}$, with a seed viability rate of $90 \%$. Morphological characteristics of fruits and seeds of Cucurbita ficifolia, C. moschata, C. maxima, Cucurbita sp., a local variety known as "loche", and Lagenaria siceraria, were evaluated. The Cucurbita accesions showed variability in fruit shape, size and colour, and in seed size and shape. Lagenaria accesions stood out by their variability in fruit shape and size, in which the "calabazos", "lapas", "mates" and "checos" fruit types, and many intermediate shapes, were recognized. The possibility that the genotypes "chuyán" and "loche", traditionally classified as $C$. moschata, could be sub-species is discussed. It is concluded that the north coast of Perú is a center of high morphological diversity of $C$. moschata and L. siceraria, while the Andean region of Cajamarca and Piura is the diversity center for C. ficifolia.

Index words: Cucurbita ficifolia, C. moschata, Lagenaria siceraria, morphological descriptors, morphological diversity.

\section{INTRODUCCIÓN}

La familia Cucurbitaceae conforma un importante grupo de plantas, mayormente tropicales, con 90 a 130 géneros y 750 a 1300 especies, muchas de ellas muy comunes y ampliamente utilizadas en la alimentación. Cinco de estas especies: Cucurbita argyrosperma Huber, C. ficifolia Bouché, C. moschata (Duchesne ex Lam.) Duchesne ex Poiret, C. maxima Duchesne ex Poiret, and C. pepo L., se domesticaron en el Nuevo Mundo y por miles de años se cultivaron o utilizaron por los aborígenes americanos (Lira y Montes, 1994).

La importancia de las cucurbitáceas no es solamente en el ámbito alimenticio sino también en el ámbito medicinal puesto que lectinas aisladas de Momordica charantia, una cucurbitaceae silvestre, inhibieron a la transcriptasa reversa HIV-1 (Wang y Ng, 2001); asimismo, dos proteínas, MRK29 (Jiratchariyakul et al., 2001) y ginkbilobina (Wang y Ng, 2000), se aislaron de frutos de $M$. charantia y de semillas de Ginkgo biloba, respectivamente, y ambos compuestos inhiben a la transcriptasa reversa HIV-1; sin embargo, MRK29 fue cien veces más activa (Cos et al., 2008).

Debido a la importancia que tienen varias especies de cucurbitáceas en la alimentación de vastos sectores de la población mundial, se les ha considerado de alta prioridad en la conservación de sus recursos genéticos (IBPGR, 1981; Esquinas-Alcázar y Gulick, 1983; Lira, 1995). Así lo demuestran varios trabajos: los realizados sobre colecta de germoplasma de cucurbitáceas en Cuba (Hammer et al., 1991); los estudios de diversidad genética de germoplasma de zapallo, Cucurbita moschata (Montes et al., 2004) y sobre caracterización molecular de introducciones nativas (Restrepo et al., 2008) en Colombia; la comparación morfológica de frutos y semillas de auyama, C. moschata (Cáseres et al., 2010) en Venezuela; las evaluaciones sobre características morfológicas de uso potencial como marcadores de especies de C. moschata y C. argyrosperma (Wessel-Beaver et al., 2004) en Puerto Rico, y la colecta y caracterización 
de germoplasma de Lagenaria siceraria (calabaza) (Yetişir et al., 2008), la evaluación del potencial de L. siceraria para ser utilizado como patrón en la injertación de yemas de $\mathrm{Ci}$ trullus lanatus (sandía) (Karaca et al., 2012), y las determinaciones de la diversidad genética de germoplasma de $C$. lanatus realizadas en Turquía (Solmaz y Sari, 2009; Uluturk et al., 2011).

Entre los trabajos más relevantes sobre evaluación morfológica se tiene el de Montes et al. (2004), quienes en 133 accesiones de C. moschata colectadas en nueve departamentos de Colombia observaron que todos los caracteres evaluados, como tamaño, forma y color de fruto, sumados a los descriptores de pubescencia de la hoja, días a floración femenina, espesor de pulpa, tamaño de la semilla, entre otros, presentaron amplio rango de valores, lo cual corrobora la gran variabilidad observada en la colección.

En el Perú se han reportado alrededor de 27 géneros y 110 especies (Brako y Zarucchi, 1993; Ulloa Ulloa et al., 2004), todos bejucos y lianas; asimismo, se ha informado la presencia de 23 especies endémicas representadas en siete géneros, además del género Guraniopsis endémico del Perú (León, 2006). También se cuenta con algunos trabajos taxonómicos y fitogeográficos sobre la familia Cucurbitaceae, en especial los de Weberbauer (1945) y Soukup (1970), e incluso sobre el comportamiento de algunas especies como flora invasora en los cultivos de la costa (Sagástegui, 1973; Sagástegui y Leiva, 1993). Sin embargo, referente a exploración y colecta de germoplasma en el Perú solamente se cuenta con el trabajo de Valega et al. (2004), donde enfatizan aspectos de distribución de Cucurbita, pero poco acerca de su caracterización morfológica.

Por estas razones se desarrolló el presente trabajo cuyo objetivo principal fue la caracterización morfológica de frutos y semillas de algunas cucurbitáceas del norte del Perú.

\section{MATERIALES Y MÉTODOS}

La exploración y colecta de germoplasma de cucurbitáceas se realizó entre julio de 1988 a noviembre de 1989 en el norte del Perú, en las regiones de Tumbes, Piura, Lambayeque y Cajamarca (Figura 1), con una extensión de 88 $079 \mathrm{~km}^{2}$, que corresponde a $6.85 \%$ del territorio nacional (1 $285216 \mathrm{~km}^{2}$ ). Las regiones exploradas comprendieron tanto a tierras bajas costaneras, muy próximas al Océano Pacífico, así como a zonas altoandinas hasta los $2500 \mathrm{~m}$ de altitud. La región Tumbes, frontera con el Ecuador, ocupa el sector más septentrional de la costa peruana; la región Piura abarca una extensa llanura agrícola, así como una no muy amplia zona andina; la región Lambayeque comprende una extensa planicie costera, en tanto que la región altoandina es reducida; y la región Cajamarca es absoluta- mente altoandina, con un relieve accidentado y complejo.

La estrategia del trabajo consistió en explorar sistemáticamente y luego colectar el germoplasma de cucurbitáceas en áreas de alta diversidad en el norte del Perú. Las accesiones, consistentes en frutos y semillas, se obtuvieron directamente de los campos de cultivo y del agricultor, aunque también se recurrió a muestras del mercado local y ferias agropecuarias, para configurar un amplio rango de variabilidad. La mayoría de las accesiones corresponden a genotipos ancestrales, con muy pocos genotipos mejorados y comerciales. Por lo general, la semilla madura se obtuvo de 3 a 5 frutos colectados en plantas diferentes.

Los datos de colecta se consignaron en Fichas Pasaporte elaboradas por IPGRI (International Plant Genetic Resources Institute) (ex IBPGR/FAO), que incluyeron los siguientes datos de cada accesión: geográficos, prácticas de cultivo, edáficos, topográficos, entre otros. Adicionalmente, en la caracterización de frutos y semillas de las accesiones se utilizaron descriptores (ECPGR, 2008), complementados con los modelos de caracterización elaborados para Lagenaria siceraria (calabaza) (Bioversity International for Cucurbitaceae, 2007; Yetişir et al., 2008) y Citrullus lanatus (sandía) (Solmaz y Sari, 2009). Sin embargo, fue necesario introducir varias modificaciones y agregar variables adicionales, como la forma de las semillas en $L$. siceraria y diversas especificaciones en el tamaño de las semillas de todas las especies de Cucurbita estudiadas, entre otras.

La mayoría de los datos consignados se obtuvieron directamente de la colección de Cucurbita y Lagenaria, y únicamente los datos de semillas se tomaron de la colección élite o núcleo, consistente de 5 a 10 muestras. Las clases por variable evaluada se expresaron en porcentaje del total de frutos caracterizados. El diámetro polar se tomó desde el punto de inserción del pedúnculo floral hasta el extremo posterior; asimismo, numerosos frutos presentaron manchas longitudinales angostas a las que se denominó "jaspes". En el análisis estadístico para el procesamiento de los datos de semilla se utilizó el programa Statistical Package for the Social Sciences (SPSS, 2009), mediante análisis de varianza y pruebas múltiples de medias (Tukey).

La cantidad de semillas colectadas por cada accesión fue dividida en dos partes iguales. Una parte se mantiene desde 1988 en el Banco de Germoplasma de la Universidad Nacional Pedro Ruiz Gallo de Lambayeque (Perú), en condiciones de refrigeración a $10^{\circ} \mathrm{C}$ de temperatura y una viabilidad germinativa de $90 \%$; y la otra parte fue enviada al IPGRI.

\section{RESULTADOS Y DISCUSIÓN}

El germoplasma colectado estuvo conformado por 8 


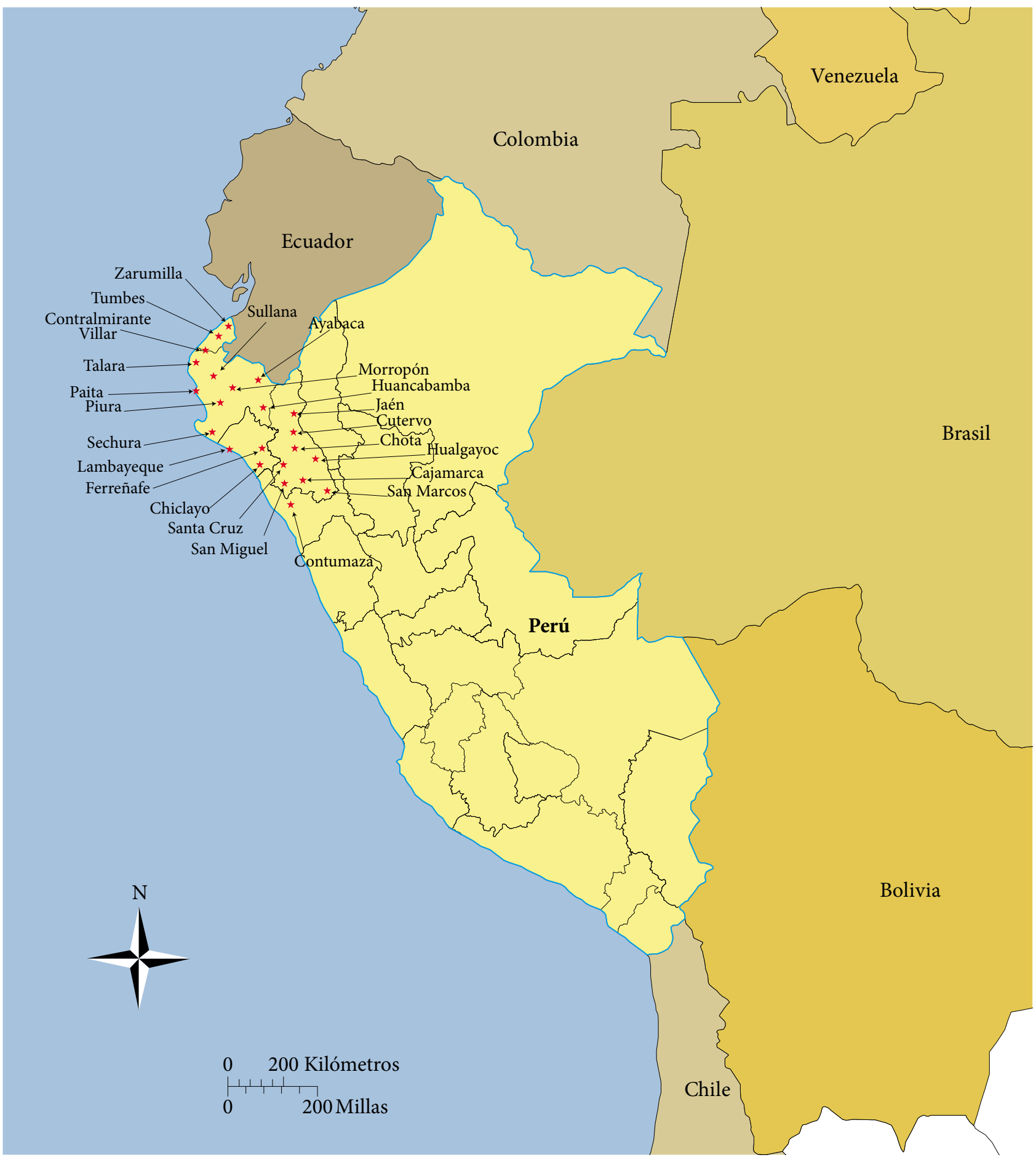

Figura 1. Sitios de colecta de germoplasma de cucurbitáceas en el norte del Perú. 
géneros y 14 especies, de las cuales nueve fueron nativas y cinco introducidas (Citrullus lanatus, Cucumis anguria, C. dipsaceus, Lagenaria siceraria y Momordica charantia) (Cuadro 1). La región con mayor número de accesiones fue Lambayeque, hecho que pudo estar influenciado por la mayor accesibilidad a las áreas de colecta. A continuación se muestran las características de frutos y semillas de algunas especies representativas del banco de germoplasma.

\section{Cucurbita ficifolia Bouché}

Nombre común: "Chiuche”, "chiclayo", "zambo", "zambomba”.

Se observó una gran diversidad morfológica entre las accesiones colectadas, sobre todo en la forma, tamaño y color de frutos y semillas (Cuadro 2 y Figura 2a). En tamaño del fruto maduro predominaron el mediano $(21$ a $30 \mathrm{~cm})$ y el grande (31 a $40 \mathrm{~cm})$, con 36 y $42 \%$, respectivamente. En la forma predominó ampliamente la cilíndrica con $64 \%$. El color principal de la cáscara varió desde crema y blanco hasta verde, y el secundario fue mayormente blanco o con jaspes sobre los frutos verdes. La textura suave o lisa de la cáscara predominó sobre la rugosa. Por su parte, la semilla fue piriforme con prevalencia del tamaño mediano (16 a $20 \mathrm{~mm}$ ) con $70 \%$ de color negro (66 \%); el mismo color se mantuvo en el borde de la semilla, y éste fue mayormente liso o uniforme (66 \%). Las medias de longitud y ancho de la semilla fueron 20.1 y $11.7 \mathrm{~mm}$, con coeficientes de variación $(\mathrm{CV})$ de 9.1 y $10.8 \%$, respectivamente, en tanto que el peso fue $25.2 \mathrm{~g}$ (Cuadro 3).

La literatura no registra trabajos sobre evaluación morfológica de germoplasma de C. ficifolia, y solamente se encontró el trabajo de Mostacero et al. (2002) quienes informaron que las plantas varían significativamente en los frutos, con formas elipsoidales o globosas, exteriormente de color blanco o verde amarillento con manchas longitudinales irregulares verdes y pulpa blanca o transparente, que pesan más de $20 \mathrm{~kg}$. Esta información se asemeja con los datos morfológicos presentados en este trabajo. En una recolección de especies hortícolas realizada en el Ecuador se colectaron 15 muestras de C. ficifolia ("zambo"), entre altitudes de 900 a $2800 \mathrm{~m}$, especie que se utiliza como verdura, confitura y forraje (Nuez et al., 1993); sin embargo, ninguna característica morfológica o agronómica fue reportada.

Estudios realizados en México permiten concluir que la variación genética es muy amplia, principalmente en la forma, tamaño y coloración del fruto, así como cantidad de semilla producida, en C. pepo y C. moshata (Montes, 1991), pero en un nivel inferior en C. maxima y C. ficifolia (Hernández, 1978). Asimismo, estudios isoenzimáticos revelaron escasa diferenciación genética de C. argyrosperma, $C$. ficifolia y C. maxima (Andres, 1990; Decker, 1988; DeckerWalters et al., 1990). Recientemente, el uso de marcadores moleculares tipo RAPD, en cuatro especies americanas cultivadas de Cucurbita, indicó reducida variabilidad genética dentro de cada especie, con $14 \%$ en C. ficifolia como el

Cuadro 1. Cucurbitáceas colectadas en el norte del Perú.

\begin{tabular}{|c|c|c|c|c|}
\hline \multirow{2}{*}{ Especies } & \multicolumn{4}{|c|}{ Regiones de Colecta } \\
\hline & Cajamarca & Lambayeque & Piura & Tumbes \\
\hline Citrullus lanatus & & 1 & & \\
\hline Cucumis anguria & 1 & & & \\
\hline C. dipsaceus & & 1 & & \\
\hline${ }^{\dagger}$ Cucurbita ficifolia & 29 & & 11 & \\
\hline${ }^{\dagger}$ C. maxima & & 13 & 8 & \\
\hline${ }^{\dagger}$ C. moschata (Zapallo) & 9 & 46 & 20 & 6 \\
\hline${ }^{\dagger}$ C. moschata (Chuyán) & 5 & & & \\
\hline${ }^{\dagger}$ Cucurbita sp. (Loche) & & 5 & & \\
\hline${ }^{\dagger}$ Cyclanthera brachybotrys & 1 & & & \\
\hline${ }^{\dagger}$ C. pedata & 9 & 2 & & \\
\hline Lagenaria siceraria & & 30 & 2 & \\
\hline${ }^{\dagger}$ Luffa operculata & 1 & & & \\
\hline Momordica charantia & 1 & & & \\
\hline${ }^{\dagger}$ Sicana odorifera & & 1 & & \\
\hline Total & 56 & 99 & 41 & 6 \\
\hline
\end{tabular}


Cuadro 2. Lista de descriptores mínimos para las especies cultivadas Cucurbita ficifolia ("chiuche"), C. moschata ("zapallo"), C. moschata ("chuyán") y Cucurbita sp. ("loche"). Observaciones realizadas en un mínimo de 10 frutos y semillas (ECPGR, 2008; Yetişir et al., 2008).

\begin{tabular}{|c|c|c|c|c|c|c|}
\hline \multirow[t]{2}{*}{ Variable } & \multirow[t]{2}{*}{ Puntaje } & \multirow[t]{2}{*}{ Clase } & $\begin{array}{l}\text { Cucurbita ficifolia } \\
\text { ("Chiuche”) }\end{array}$ & $\begin{array}{c}\text { Cucurbita moschata } \\
\text { ("Zapallo") }\end{array}$ & $\begin{array}{c}\text { Cucurbita moschata } \\
\text { (“Chuyán") }\end{array}$ & $\begin{array}{l}\text { Cucurbita sp. } \\
\text { ("Loche”) }\end{array}$ \\
\hline & & & \multicolumn{4}{|c|}{ No/(\%) } \\
\hline \multirow[t]{4}{*}{$\begin{array}{l}\text { Frutos } \\
\text { Tamaño } \\
\text { (Ø polar) }\end{array}$} & 3 & $\begin{array}{l}\text { Pequeño } \\
(\leq 20 \mathrm{~cm})\end{array}$ & $4(11.1)$ & $22(28.6)$ & $5(100.0)$ & $1(20.0)$ \\
\hline & 5 & $\begin{array}{c}\text { Medio } \\
(21 \mathrm{a} 30 \mathrm{~cm})\end{array}$ & $13(36.1)$ & $34(44.2)$ & & $3(60.0)$ \\
\hline & 7 & $\begin{array}{c}\text { Grande } \\
(31 \mathrm{a} 40 \mathrm{~cm})\end{array}$ & $15(41.7)$ & $18(23.4)$ & & $1(20.0)$ \\
\hline & 9 & $\begin{array}{l}\text { Muy grande } \\
(\geq 41 \mathrm{~cm})\end{array}$ & $4(11.1)$ & $3(3.8)$ & & \\
\hline \multirow[t]{15}{*}{ Forma } & 1 & Globular & $5(13.9)$ & $13(14.6)$ & $1(20.0)$ & \\
\hline & 2 & Aplanado & $3(8.3)$ & $14(16.9)$ & $2(40.0)$ & \\
\hline & 3 & Discoidal & & & & \\
\hline & 4 & Cilíndrico & $23(63.9)$ & $17(20.4)$ & & \\
\hline & 5 & Elíptico & $2(5.5)$ & $6(7.4)$ & & \\
\hline & 6 & Acorazonado & & & & \\
\hline & 7 & Piriformes & $2(5.5)$ & $34(40.7)$ & $2(40.0)$ & $5(100.0)$ \\
\hline & 8 & $\begin{array}{l}\text { Dumbbell } \\
\text { (con cuello) }\end{array}$ & & & & \\
\hline & 9 & Elongado & $1(2.9)$ & & & \\
\hline & 10 & Turbinado sup. & & & & \\
\hline & 11 & Coronado & & & & \\
\hline & 12 & Turbinado inf. & & & & \\
\hline & 13 & Curvado & & & & \\
\hline & 14 & Cuello doblado & & & & \\
\hline & 99 & Otros & & & & \\
\hline \multirow[t]{12}{*}{$\begin{array}{l}\text { Color de la } \\
\text { cáscara }\end{array}$} & 1 & Blanco & $11(30.5)$ & $2(2.6)$ & & \\
\hline & 2 & Verde & $23(63.9)$ & $54(71.1)$ & $5(100.0)$ & $3(60.0)$ \\
\hline & 3 & Azul & & & & \\
\hline & 4 & Crema & $2(5.6)$ & & & \\
\hline & 5 & Amarillo & & $10(13.2)$ & & $1(20.0)$ \\
\hline & 6 & Anaranjado & & $9(11.8)$ & & $1(20.0)$ \\
\hline & 7 & Rojo & & & & \\
\hline & 8 & Rosado & & $1(1.3)$ & & \\
\hline & 9 & Marrón & & & & \\
\hline & 10 & Gris & & & & \\
\hline & 11 & Negro & & & & \\
\hline & 99 & $\begin{array}{l}\text { Otro (verde con } \\
\text { jaspes blancos) }\end{array}$ & & & & \\
\hline
\end{tabular}


Cuadro 2. Continuación.

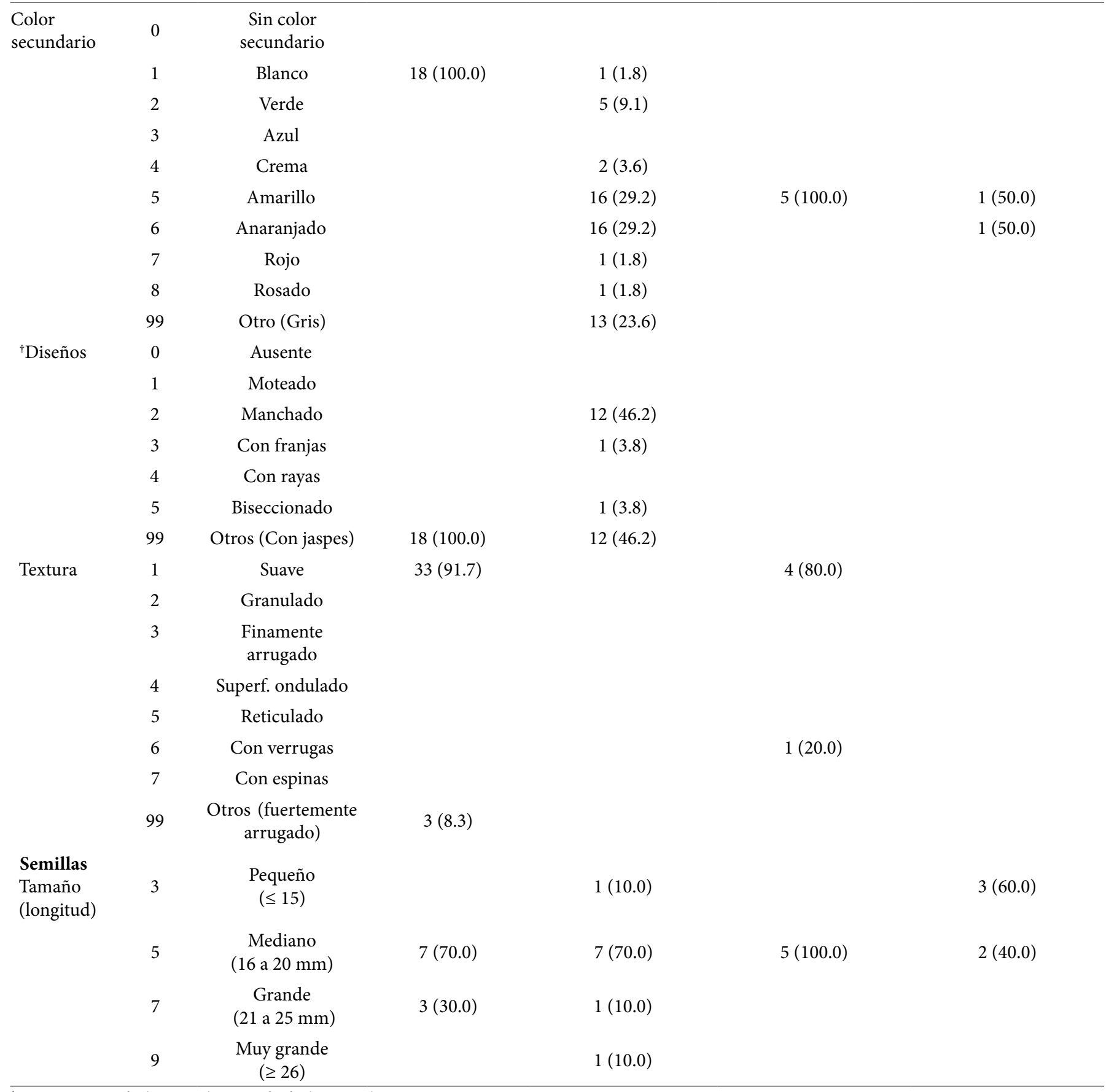

${ }^{\dagger}$ Diseños: Moteado $(<0.5 \mathrm{~cm})$ y Manchado $(\geq 0.5 \mathrm{~cm})$. 


\section{Cuadro 2. Continuación.}

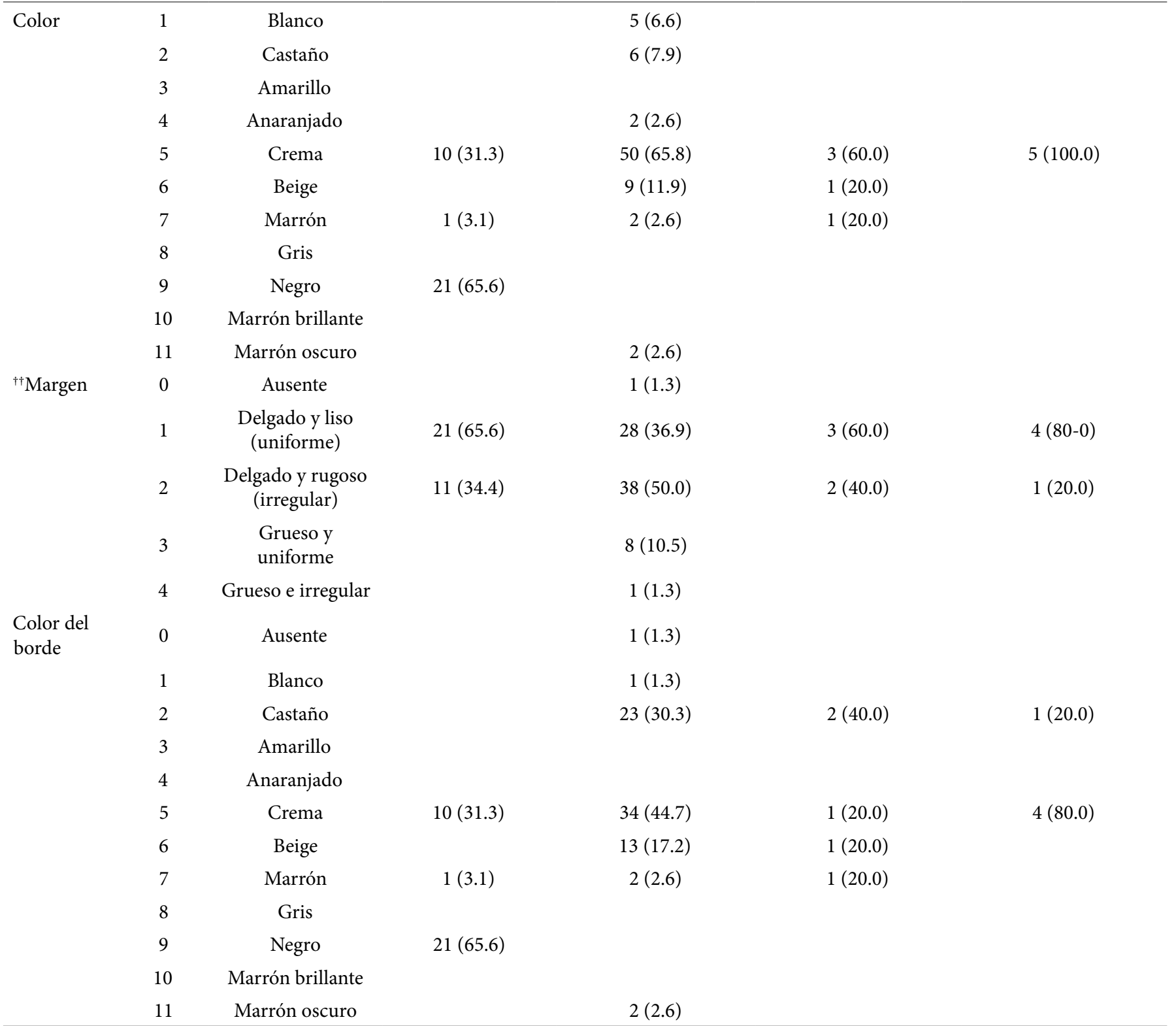

${ }^{\dagger \dagger}$ Margen de la semilla ( $\leq 2 \mathrm{~mm}$, delgado; $\mathrm{y}>2 \mathrm{~mm}$, grueso). 

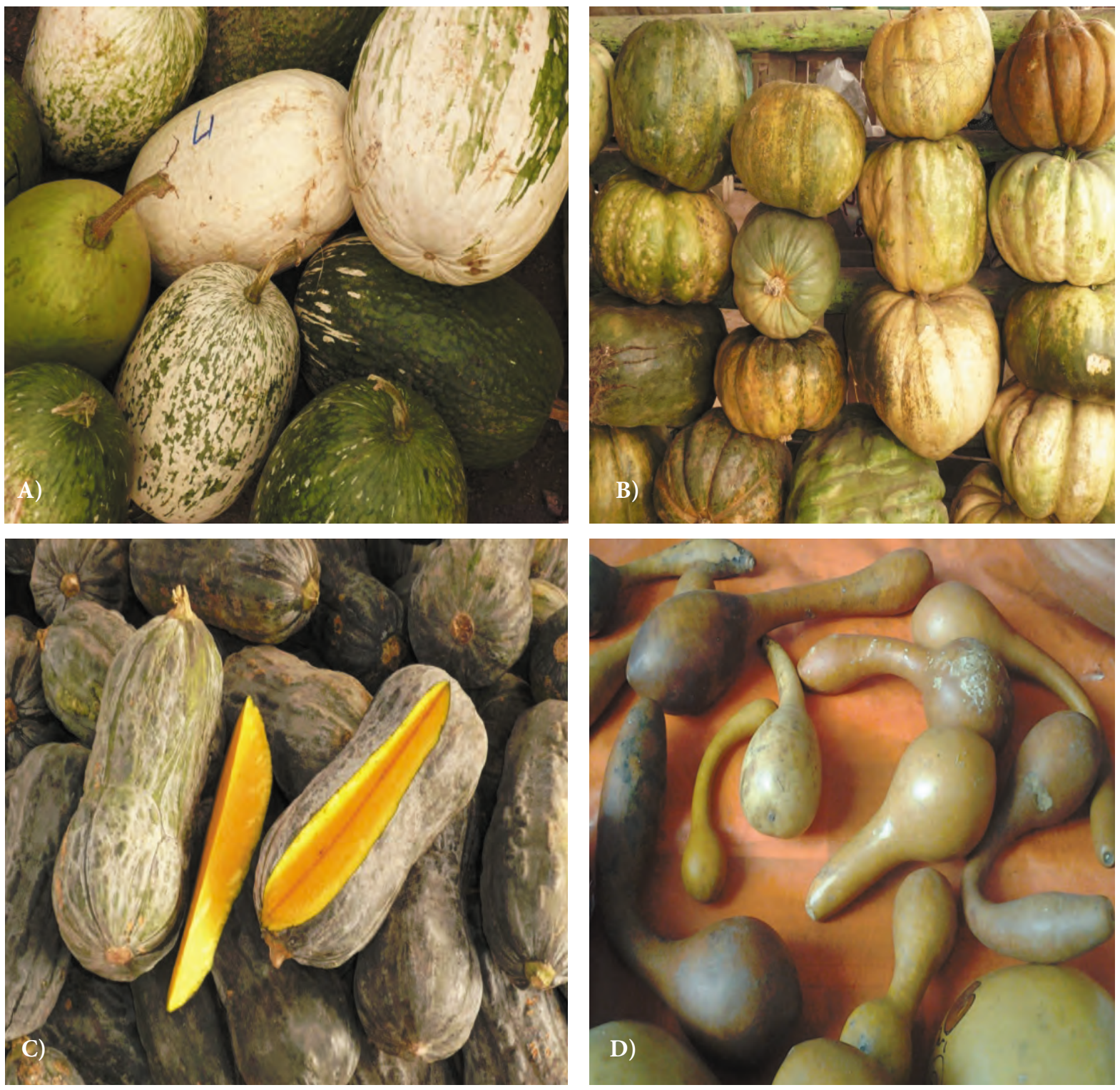

Figura 2. Muestra de la variación morfológica en frutos de Cucurbita spp. A) "Chiuche” (C. ficifolia); B) “Zapallo" (C. moschata); C) "Loche" (Cucurbita sp.) y D) “Calabazo" (Lagenaria siceraria), utilizado para dar masaje, obtenido en un mercado de la ciudad de Chiclayo (Lambayeque).

valor más bajo, y $37 \%$ en C. moschata como el valor más alto. Aun cuando las especies se encontraban altamente diferenciadas, $C$. ficifolia resultó la más alejada con un coeficiente de identidad de Nei de alrededor de 0.5 (Cerón et al., 2010), el cual determina el porcentaje de loci polimórficos (Nei, 1973). Es posible que estos resultados obedezcan a que en México no ocurre tanta diversidad morfológica de C. ficifolia como en los valles andinos del Perú, como aquí se evidencia principalmente en cuanto a forma, tamaño y color de frutos y semillas.

\section{Cucurbita maxima Duchesne}

Nombre común: "Zapallo", "macre”, "fuque”, "zapallo loche”.

Aun cuando en el presente trabajo no se evaluaron de manera sistemática las características morfológicas de frutos, se observó que los de las variedades de la costa pesaron entre 50 a $60 \mathrm{~kg}$, con cáscara lisa, hendiduras longitudinales, color verde oscuro hasta verde claro y aspecto lustroso. Las variedades altoandinas, cultivadas entre 1500 a $2800 \mathrm{~m}$ de altitud, registraron frutos con un peso superior a $100 \mathrm{~kg}$, cáscara de color verde cenizo y variaciones amarillentas y 
Cuadro 3. Caracterización de semillas de varias especies de cucurbitáceas colectadas en el norte del Perú.

\begin{tabular}{|c|c|c|c|c|c|c|c|c|c|}
\hline \multirow{2}{*}{$\begin{array}{l}\text { Largo/ancho } \\
(\mathrm{mm})\end{array}$} & \multirow[t]{2}{*}{$\operatorname{Media}^{\dagger}(\mathrm{mm})$} & \multirow[t]{2}{*}{ Desv. est. } & \multirow[t]{2}{*}{ CV. (\%) } & \multicolumn{2}{|c|}{$\begin{array}{c}\text { Intervalo de } \\
\text { confianza }(95 \%)^{\dagger \dagger}\end{array}$} & \multicolumn{2}{|c|}{ Rango" } & \multirow[t]{2}{*}{$\operatorname{Peso}^{99}(\mathrm{~g})$} & \multirow[t]{2}{*}{ Forma } \\
\hline & & & & LI & LS & $\mathrm{Mi}$ & $\mathrm{Mx}$ & & \\
\hline \multicolumn{10}{|l|}{ C. ficifolia } \\
\hline Largo $(\mathrm{mm})$ & 20.1 & 1.810 & 9.01 & 19.7 & 20.4 & 17.0 & 25.0 & 25.24 & Piriforme \\
\hline Ancho (mm) & 11.7 & 1.209 & 10.37 & 11.4 & 11.9 & 8.0 & 14.0 & & \\
\hline \multicolumn{10}{|l|}{ C. maxima } \\
\hline Largo (mm) & 20.1 & 5.055 & 25.22 & 19.1 & 21.1 & 12.0 & 32.0 & 34.46 & Piriforme \\
\hline Ancho (mm) & 10.8 & 3.423 & 31.60 & 10.5 & 11.5 & 6.0 & 19.0 & & \\
\hline \multicolumn{10}{|l|}{ C. moschata } \\
\hline Largo (mm) & 16.9 & 1.058 & 6.24 & 16.6 & 17.2 & 15.0 & 19.0 & 11.28 & Piriforme \\
\hline Ancho (mm) & 8.5 & 0.735 & 8.64 & 8.29 & 8.7 & 7.0 & 10.0 & & \\
\hline \multicolumn{10}{|c|}{ C. moschata (Chuyán) } \\
\hline Largo $(\mathrm{mm})$ & 17.6 & 1.383 & 7.8 & 17.2 & 18.0 & 15.0 & 20.0 & 8.03 & Piriforme \\
\hline Ancho (mm) & 8.8 & 1.017 & 11.50 & 8.6 & 9.1 & 7.0 & 11.0 & & \\
\hline \multicolumn{10}{|c|}{ Cucurbita sp. (Loche) } \\
\hline Largo $(\mathrm{mm})$ & 15.1 & 2.370 & 15.67 & 14.5 & 15.8 & 12.0 & 20.0 & 6.42 & Piriforme \\
\hline Ancho (mm) & 8.3 & 2.221 & 26.88 & 7.6 & 8.9 & 6.0 & 13.0 & & \\
\hline \multicolumn{10}{|l|}{ L. siceraria } \\
\hline Largo $(\mathrm{mm})$ & 14.1 & 2.873 & 20.40 & 13.5 & 14.7 & 9.0 & 18.0 & 10.63 & Escudete y \\
\hline Ancho (mm) & 7.5 & 1.431 & 19.20 & 7.2 & 7.7 & 6.0 & 12.0 & & Piriforme \\
\hline
\end{tabular}

${ }^{\dagger}$ Promedio de 10 accesiones; ${ }^{\dagger \dagger} \mathrm{LI}=$ límite inferior; $\mathrm{LS}=$ líimite superior; ${ }^{9} \mathrm{Mi}=$ mínimo; $\mathrm{Mx}=$ máximo; "Promedio de 100 semillas.

anaranjadas. Las semillas piriformes fueron muy variables en tamaño y color, con medias de ancho y longitud de 10.8 y $20.1 \mathrm{~mm}$, y coeficientes de variación (CV) de 25.2 y 31.6 $\%$, respectivamente, en tanto que el peso fue $34.5 \mathrm{~g}$ (Cuadro 3). Estos CV significativamente altos se atribuyen a la alta variación en el tamaño de la semilla.

\section{Cucurbita moschata (Duch. ex Lam.) Duch. ex Poiret} Nombre común: Los mismos asignados a C. maxima.

$\mathrm{Al}$ igual que para el "chiuche", entre las accesiones colectadas en el norte del Perú se observó una gran diversidad morfológica, variación que fue mayor en la forma, tamaño $y$ color de frutos y semillas (Cuadro 2 y Figura 2b). El tamaño del fruto más abundante correspondió al medio (21 a $30 \mathrm{~cm}$ ) con $44 \%$. La forma fue también muy variable, pero con predominio de las formas piriforme y cilíndrica, con 41 y $20 \%$, respectivamente. El color principal de la cáscara fue verde (oscuro y claro) con $71 \%$, y el secundario fue amarillo, anaranjado y gris, en la mayoría de los casos con jaspes y manchas irregularmente circulares y dispersas; la superficie fue lisa, uniforme o con hendiduras longitudinales poco profundas, y sólo en unos casos era muy rugosa; pulpa amarilla o anaranjada; y semillas de varios colores; fruto de tamaño variable, desde accesiones con menos de $1 \mathrm{~kg}$ hasta unas con más de $10 \mathrm{~kg}$. La semilla era piriforme de tamaño mediano (16 a $20 \mathrm{~mm}$ ) en $70 \%$ de los casos; su color fue mayoritariamente crema (66\%); con borde delgado y rugoso (50 \%) de color crema (45 \%). Las medias de longitud y ancho de la semilla fueron 16.9 y $8.5 \mathrm{~mm}$, con CV de 6.2 y $8.6 \%$, respectivamente, en tanto que el peso fue $11.3 \mathrm{~g}$ (Cuadro 3).

Por otro lado, en los valles templados de la sierra se cultiva una variedad de "zapallo" conocida como "zapallo cushi" o "chuyán" que también es muy polimorfo y se usa como alimento de cerdos. El tamaño del fruto maduro fue siempre pequeño $(\leq 20 \mathrm{~cm})$, cuya forma varió entre aplanado (40\%) y piriforme (40\%). El color primario de la cáscara fue verde con amarillo secundario en todos los casos, de textura mayormente suave $(80 \%)$. La semilla fue piriforme, de tamaño $100 \%$ mediano (16 a $20 \mathrm{~mm}$ ) y de color principalmente crema (60\%), con borde delgado y liso (60\%) o delgado y rugoso (40\%), de color predominantemente castaño (40 \%). Las medias de longitud y ancho de la semilla fueron 17.6 y $8.8 \mathrm{~mm}$, con CV de 7.8 y $11.5 \%$, respectivamente; su peso fue $8.0 \mathrm{~g}$ (Cuadro 3 ). 
"Zapallo" (C. moschata) es otra de las cinco especies del género Cucurbita domesticadas en eventos independientes o simultáneos en Mesoamérica y norte de Sudamérica (Sanjur et al., 2002; Ferriol et al., 2004). Esta especie produce frutos muy variables en forma, tamaño, color, grosor del mesocarpio y epicarpio, y en morfología de las semillas (Lira, 1995; Azurdia, 1999). Esta diversidad ha sido poco estudiada a pesar de que algunas características pudieran beneficiar la calidad culinaria de los frutos (Paris, 1996). Un tipo pequeño de C. moshata, denominado "chuyán" o "zapallo cushi”, que algunos taxónomos locales consideran una posible subespecie o quizás especie, se reporta para la sierra norte del Perú. En su estudio, Montes et al. (2004) informaron que la longitud del fruto registró una media de $25.11 \mathrm{~cm}$, que guarda relación con los datos obtenidos en el trabajo que aquí presenta puesto que $36.1 \%$ de los frutos de la colección peruana correspondió al tamaño medio (21 a $30 \mathrm{~cm}$ ). En lo referente al peso de 100 semillas, estos autores reportaron un valor de $12.18 \mathrm{~g}$, muy similar a lo obtenido en el presente trabajo (11.28 g). Desafortunadamente, el trabajo de Montes et al. (2004) no precisó las características de esta variación.

Al comparar frutos y semillas de "auyama” (C. moschata) de las formas lageniformes (cuello de cisne) y elípticos a oblongos, colectados en Guanare (Venezuela), Cáseres et al. (2010) determinaron que los primeros eran de mayor longitud y ancho que los segundos, aunque estos últimos fueron ligeramente más pesados. En cuanto a las semillas, las de los lageniformes fueron de mayor tamaño, pero de menor peso respecto de los elípticos a oblongos. Los resultados aquí obtenidos sobre fruto fueron semejantes, pero no en lo referente a semillas. Aun cuando la forma del fruto es fundamental para el aprovechamiento del mesocarpio, cuyas formas achatadas se prefieren para consumo humano como verdura o dulces mientras que las formas elípticas que son utilizadas en la alimentación animal (Paris, 1966), en el Perú, con excepción del tipo andino denominado "chuyán", que es achatado y utilizado para alimentar cerdos, los demás tipos de calabaza se aprovechan en alimentación humana.

En concordancia con la diversidad morfoagronómica de C. moschata, determinada en el presente trabajo con accesiones peruanas, así como en los trabajos de Montes et al. (2004) en Colombia y de Cáseres et al. (2010) en Venezuela, la alta diversidad genética de esta especie fue corroborada de manera indirecta mediante el polimorfismo en la longitud de fragmentos amplificados (AFLP) de 121 introducciones de C. moschata provenientes de ocho departamentos de Colombia, en las que se determinó que la mayoría de la variación genética era consecuencia de la variación entre individuos dentro de cada departamento (Restrepo y Vallejo, 2008). No obstante la innegable utilidad de las técni- cas moleculares, todavía resulta válido el empleo de otras características morfológicas de diferenciación como el uso de los estambres de las flores masculinas para la caracterización de híbridos intra e interespecíficos de C. moschata y C. argyrosperma (Wessel-Beaver et al., 2004), toda vez que otras características morfológicas establecidas para Cucurbita cultivadas no han sido satisfactorias para diferenciar eficientemente a los taxas (Lira-Saade et al., 1995).

\section{Cucurbita sp.}

Nombre común: "Loche”, "calabaza”, "zapallo criollo”.

"Loche" es una variedad muy apreciada en Lambayeque, que presenta frutos pequeños, alargados, cuello poco pronunciado y superficie muy rugosa; su pulpa es anaranjada, compacta y en la madurez desarrolla una pequeña cavidad en el extremo ensanchado donde se encuentran algunas semillas mayormente estériles (Figura 2c). La propagación comercial es vegetativa mediante esquejes. El fruto de esta especie es $60 \%$ de tamaño medio $(21$ a $30 \mathrm{~cm})$, piriforme, con color predominante de la cáscara $60 \%$ verde y el secundario $50 \%$ amarillo y $50 \%$ anaranjado. La semilla piriforme es de tamaño $60 \%$ pequeño $(\leq 15 \mathrm{~mm}$ ), de color $100 \%$ crema, con borde mayormente delgado y liso (80 \%) de color crema ( $80 \%$ ) (Cuadro 2). Las medias de longitud y ancho de la semilla son 15.1 y $8.3 \mathrm{~mm}$, con CV de 15.7 y $26.9 \%$, respectivamente, valores relativamente altos; su peso es de $6.4 \mathrm{~g}$ (Cuadro 3).

El "loche" es muy utilizado en la preparación del platillo denominado cabrito o chivo estofado, por el agradable sabor que le confiere. Es posible que se trate de una variedad local de C. moschata, o de un híbrido entre ésta y C. maxima. Existen varias razones para fundamentar esta hipótesis de hibridación: el "loche" tiene frutos y semillas muy semejante a C. moschata, produce escasa semilla sexual o ninguna, la propagación comercial se hace por esquejes, y cuando el cultivo es por semilla sexual se obtienen frutos segregantes llamados "zapallo-loche", es decir, "loche" con características de "zapallo" en forma, tamaño e incluso sabor, por lo que se considera una adulteración del verdadero "loche". Todo esto configuraría su naturaleza híbrida, pero es necesario efectuar trabajos morfológicos y genéticos detallados. Desafortunadamente el trabajo de Cerón et al. (2010) sobre diversidad genética entre especies de Cucurbita americanas no incluyó a C. maxima, y aunque el coeficiente de identidad de Nei es indicador de una gran proximidad genética entre C. moschata y C. agyrosperma, esta última especie no se encuentra en el Perú.

\section{Lagenaria ciceraria (Molina) Stanley}

Nombre común: "Mate”, "calabaza”, "lapa”, “cojudito", "checo", "checo coquero". 
En el Cuadro 4 se muestra la amplia diversidad morfológica entre los diversos genotipos de "calabazos" y "lapas" colectados en la costa norte del Perú, variación que fue mayor en el tamaño y forma de frutos y semillas. En el tamaño del fruto prevaleció el pequeño (69.9\%), piriforme de cuello recto con dimensiones que oscilan desde $7.5 \mathrm{~cm}$ de altura y $3 \mathrm{~cm}$ de diámetro ("checo coquero") hasta grandes y aplanados de $20 \mathrm{~cm}$ de altura y $55 \mathrm{~cm}$ de diámetro ("lapa"). En cuanto a forma del fruto prevaleció la piriforme con cuello recto corto o largo, y ligeramente curvado $(60 \%)$. Destacó un genotipo con fruto en forma de guitarra (panduriforme), que no ha sido reportado en otros descriptores (ECPGR, 2008; Yetişir et al., 2008). Referente al color predominante de la cáscara, dominó el marrón (93.4 \%) en varios tonos. Las medias de longitud y ancho de la semilla fueron 14.1 y $7.5 \mathrm{~mm}$, con CV de 20.4 y $19.2 \%$, respectivamente, en tanto que el peso fue $10.6 \mathrm{~g}$ (Cuadro 3). En la Figura $2 \mathrm{~d}$ se muestran calabazos pequeños utilizados para masajes corporales.

Las descripciones convencionales indican que los frutos son de tamaño variable y van desde muy pequeños de $5 \mathrm{~cm}$ de diámetro, hasta muy grandes de más de $1 \mathrm{~m}$ de largo; desde forma redonda como los "mates" y "lapas" hasta la de botella como los "calabazos", con múltiples formas intermedias que han abierto una gran posibilidad de usos. Las semillas también variaron ampliamente en tamaño, forma y color. En uno de los trabajos más relevantes sobre colecta y caracterización morfológica de 182 accesiones de L. siceraria realizado en la región Mediterránea de Turquía, también se encontró una gran diversidad morfológica en la forma y tamaño de frutos, y en forma, tamaño y color de semillas, aunque Turquía no es centro de diversidad genética (Yetişir et al., 2008).

Esta variabilidad de frutos y semillas de los materiales evaluados en Turquía es tan grande como la encontrada en el norte del Perú; sin embargo, en Turquía solamente se observó semilla con la forma escudete, pero no la piriforme. La dispersión natural y artificial de L. siceraria de África hacia numerosos lugares del mundo desde hace miles de años, determinó que en algunos sitios se reconocieran subespecies como en Italia con L. siceraria ssp. siceraria y L. siceraria ssp. asiatica (Hammer et al., 1999). De ahí la necesidad de sumar la caracterización molecular a la caracterización morfológica. Con el uso de marcadores RAPD y secuenciación del ADN del cloroplasto para analizar 43 cultivares de calabazos procedentes de África, Asia y el Nuevo Mundo, se ha determinado que las accesiones y cultivares comerciales analizados son distintos y con diversa base genética (Decker-Walters et al., 2001; Decker-Walters et al., 2004). Debido a la gran diversidad de formas de frutos y semillas que presentaron los calabazos en el norte del Perú, es posible postular la existencia de subespecies.

\section{CONCLUSIONES}

Existe variabilidad genética en las especies de Cucurbita del norte del Perú expresada en la morfología de frutos y semillas, variación que es mayor entre especies y en menor proporción dentro de especies. La variabilidad morfológica más alta ocurre en Lagenaria siceraria cuyos frutos son conocidos como "calabazas", "mates", "lapas" y "checos".

\section{AGRADECIMIENTOS}

Al International Plant Genetic Resources Institute (IPGRI), por el apoyo financiero en la colecta de germoplasma; y a César Estela Campos, por haber colaborado en la colecta de germoplasma.

\section{BIBLIOGRAFÍA}

Andres T. C. (1990) Biosystematics, theories on the origin and breeding potential of Cucurbita ficifolia. In: Biology and Utilization of the Cucurbitaceae. D M Bates (ed). Comstock Publishing Associates. Ithaca, N.Y. pp:102-119.

Azurdia C. (1999) Las cucúrbitas de Guatemala. Tikalia 17:41-58.

Bioversity International for Cucurbitaceae (2007) Guidelines for the Development of Crop Descriptor Lists. Bioversity Technical Bulletin Series. Bioversity International, Rome, Italy. 72 p.

Brako L. and J. Zarucchi (1993) Catalogue of the Flowering Plants and Gymnosperms in Peru. Mongr. Missouri Botanical Garden 45.

Cáseres E., K. Piña, T. Berríos y N. Leal (2010) Comparación morfoló gica de frutos y semillas de auyama (Cucurbita moschata Duch. ex Lam.). Revista UNELLEZ de Ciencia y Tecnología 28:32-36.

Cerón L., J. P. Legaria, C. Villanueva y J. Sahagún (2010) Diversidad genética en cuatro especies mexicanas de calabaza (Cucurbita spp.). Revista Fitotecnia Mexicana 33:189-196.

Cos P, L Maes, A Vlietinck, L Pieters (2008) Plant-derived leading compounds for chemotherapy of human immunodeficiency virus (HIV) infection an update (1998-2007). Planta Medica 74:1323-1337.

Decker D. S. (1988) Origin(s), evolution, and systematics of Cucurbita pepo (Cucurbitaceae). Economic Botany 42:4-15.

Decker-Walters D. S., T. W. Walters, U. Poluszny and P. G. Kevan (1990) Genealogy and gene flow among annual domesticated species of Cucurbita. Canadian Journal of Botany 68:782-789.

Decker-Walters D. S., J. Staub, A. López-Sésé and E. Nakata (2001) Diversity in landraces and cultivars of bottle gourd (Lagenaria siceraria; Cucurbitaceae) as assessed by random amplified polymorphic DNA. Genetic Resources and Crop Evolution 48:369380.

Decker-Walters D. S., M. Wilkins-Ellert, S. Chung and J. E. Staub (2004) Discovery and genetic assessment of wild bottle gourd [Lagenaria siceraria; (Mol.) Standley; Cucurbitaceae] from Zimbabwe. Economic Botany 58:501-508.

Esquinas-Alcázar J. T. and P. J. Gulick (1983) Genetic Resources of Cucurbitaceae: A Global Report. International Board for Plant Genetic Resources Secretariat. Rome, Italy. 101 p.

ECPGR, European Cooperative Programme for Plant Genetic Resources (2008) Minimum Descriptors for Cucurbita spp., Cucumber, Melon and Watermelon. ECPGR Secretariat. 13 p.

Ferriol M., B. Picó, P. Fernández de Córdova y F. Nuez (2004) Molecular diversity of a germplasm collection of squash (Cucurbita moschata) determined by SRAP and AFLP markers. Crop Science 44:653-664.

Hammer K., M. Esquivel and E. Carmona (1991) Plant Genetic Resources in Cuba. Report of a Collecting Mission, Febraury_March 1990. Plant Genetic Resources Newsletter 86:21-27.

Hammer K., H. Knüpffer, G. Laghetti and P. Perrino (1999) Seeds from the Past. A Catalogue of Crop Germplasm in Central and North 
Cuadro 4. Lista mínima de descriptores para la especie cultivada L. siceraria. (Basado parcialmente en Bioversity International for Cucurbitáceae, 2007; Yetişir et al., 2008).

\begin{tabular}{|c|c|c|c|}
\hline Variable & Puntaje & Clase & L. siceraria $\left(\mathrm{N}^{\circ} / \%\right)$ \\
\hline \multirow{4}{*}{$\begin{array}{l}\text { Frutos } \\
\text { Tamaño ( } \varnothing \text { polar })\end{array}$} & 3 & Pequeño $(\leq 20 \mathrm{~cm})$ & $20(69.0)$ \\
\hline & 5 & $\operatorname{Medio}(21$ a $30 \mathrm{~cm})$ & $5(17.2)$ \\
\hline & 7 & Grande $(31 \mathrm{a} 40 \mathrm{~cm})$ & $2(6.9)$ \\
\hline & 9 & Muy grande $(\geq 41 \mathrm{~cm})$ & $2(6.9)$ \\
\hline \multirow[t]{15}{*}{ Forma } & 1 & Globular (Redondo) & $1(3.3)$ \\
\hline & 2 & Aplanado & $4(13.4)$ \\
\hline & 3 & Discoidal & \\
\hline & 4 & Cilíndrico & $3(10.0)$ \\
\hline & 5 & Elíptico (ovalado) & $1(3.3)$ \\
\hline & 6 & Acorazonado & \\
\hline & 7 & Piriforme & $18(60.0)$ \\
\hline & 8 & Pandurado (con cuello) & $1(3.3)$ \\
\hline & 9 & Elongado & \\
\hline & 10 & Turbinado superior & \\
\hline & 11 & Coronado & \\
\hline & 12 & Turbinado inferior & \\
\hline & 13 & Curvado & \\
\hline & 14 & Cuello doblado & $2(6.7)$ \\
\hline & 99 & Otros & \\
\hline \multirow[t]{12}{*}{ Color de la cáscara } & 1 & Blanco & \\
\hline & 2 & Verde & $1(3.3)$ \\
\hline & 3 & Azul & \\
\hline & 4 & Crema & $1(3.3)$ \\
\hline & 5 & Amarillo & \\
\hline & 6 & Anaranjado & \\
\hline & 7 & Rojo & \\
\hline & 8 & Rosado & \\
\hline & 9 & Marrón & $28(93.4)$ \\
\hline & 10 & Gris & \\
\hline & 11 & Negro & \\
\hline & 99 & Otros & \\
\hline
\end{tabular}


Cuadro 4. Continuación.

\begin{tabular}{|c|c|c|c|}
\hline \multirow{2}{*}{$\begin{array}{l}\text { Semillas } \\
\text { Forma }\end{array}$} & 3 & Escudete & $30(73.2)$ \\
\hline & 5 & Piriforme & $11(26.8)$ \\
\hline \multirow[t]{3}{*}{ Tamaño (longitud) } & 3 & Pequeño (8 a 11 mm) & $3(30.0)$ \\
\hline & 5 & Medio (12 a $15 \mathrm{~mm})$ & $3(30.0)$ \\
\hline & 7 & Grande $(>16 \mathrm{~mm})$ & $4(40.0)$ \\
\hline \multirow[t]{5}{*}{ Margen } & 0 & Ausente & $14(36.8)$ \\
\hline & 1 & Unifor. delgado (liso) & $15(39.5)$ \\
\hline & 2 & Irreg. delgado (rugoso) & $9(23.7)$ \\
\hline & 3 & Uniformemente grueso & \\
\hline & 4 & Irregularmente grueso & \\
\hline \multirow[t]{13}{*}{ Color } & 0 & Ausente & \\
\hline & 1 & Blanco & \\
\hline & 2 & Castaño (bronceado) claro & $12(31.6)$ \\
\hline & 3 & Castaño oscuro & $6(15.8)$ \\
\hline & 4 & Amarillo & \\
\hline & 5 & Anaranjado & \\
\hline & 6 & Beige & $1(2.6)$ \\
\hline & 7 & Crema & \\
\hline & 8 & Gris & \\
\hline & 9 & Marrón & $15(39.5)$ \\
\hline & 10 & Marrón brillante & $3(7.9)$ \\
\hline & 11 & Marrón oscuro & $1(2.6)$ \\
\hline & 12 & Negro & \\
\hline \multirow[t]{13}{*}{ Color del margen } & 0 & Ausente & $14(36.7)$ \\
\hline & 1 & Blanco & \\
\hline & 2 & Castaño (bronceado) claro & $8(21.1)$ \\
\hline & 3 & Castaño oscuro & \\
\hline & 4 & Amarillo & \\
\hline & 5 & Anaranjado & \\
\hline & 6 & Beige & $3(7.9)$ \\
\hline & 7 & Crema & $8(21.1)$ \\
\hline & 8 & Gris & \\
\hline & 9 & Marrón & $3(7.9)$ \\
\hline & 10 & Marrón brillante & $2(5.3)$ \\
\hline & 11 & Marrón oscuro & \\
\hline & 11 & Negro & \\
\hline
\end{tabular}


Italy. CNR, Instituto del Germoplasma, Bari. $254 \mathrm{p}$.

Hernández B G (1978) Cucurbitaceas. In: T Cervantes S (ed). Recursos Genéticos Disponibles a México. SOMEFI. Chapingo, México. pp:357-367.

IBPGR, International Board for Plant Genetic Resources (1981) Revised Priorities Among Crops and Regions. International Board for Plant Genetic Resources Secretariat. Rome, Italy. 18 p.

Jiratchariyakul W., C. Wiwat, M. Vongsakul, A. Somanabandhu, W. Leelamanit, I. Fuji, N. Suwannaroj and Y. Ebizuka (2001) HIV inhibitor from Thai bitter gourd. Planta Medica 67:350353.

Karaca F., H. Yetișir, I. Solmaz, E. Candir, S Kurt, N. Sari and Z. Güler (2012) Rootstock potential of Turkish Lagenaria siceraria germplasm for watermelon: plant growth, yield and quality. Turkish Journal Of Agriculture \& Forestry 36:167-177.

León B. (2006) Cucurbitaceae endémicas del Perú. In: El Libro Rojo de las Plantas Endémicas del Perú. B León et al. (ed). Revista Peruana de Biología 13: 271s-274s.

Lira R. (1995) Estudios Taxonómicos y Ecogeográficos de las Cucurbitaceae Latinoamericanas de Importancia Económica: Cucurbita, Sechium, Sicana y Cyclanthera. Systematic and Ecogeographic Studies on Crop Genepools. 9. International Plant Genetic Resources Institute/Instituto de Biología, Universidad Nacional Autónoma de México. Rome, Italy. 281 p.

Lira R. and S. Montes (1994) Neglected crops: 1492 from a different perspective. In: Plant Production and Protection. J E Hernando B, J León (eds). Series No. 26. FAO, Rome, Italy. pp: 63-77.

Lira-Saade R., T. C. Andres and M. Nee (1995) Capítulo 1. Cucurbita L. In: Estudios Taxonómicos y Ecogeográficos de las Cucurbitaceae Latinoamericanas de Importancia Económica. R LiraSaade (ed). International Plant Genetic Resources Institute, Rome, Italy.

Montes H. S. (1991) Calabazas (Cucurbita spp.). In: Avances en el Estudio de los Recursos Fitogenéticos de México. R Ortega P, G Palomino H, F Castillo G, V A González H, M Livera M (eds). SOMEFI. Chapingo, México. pp:239-250.

Montes R. C., C. F. A. Vallejo and G. D. Baena (2004) Diversidad genética de germoplasma colombiano de zapallo (Cucurbita moschata Duchesne ex Poir). Acta Agronómica (Palmira) 53:43-50.

Mostacero J., F .Mejía and O. Gamarra (2002) Taxonomía de las Fanerógamas Útiles del Perú. Vol. I. Consejo Nacional de Ciencia y Tecnología (CONCYTEC). Trujillo, Perú. 667 p.

Nei M. (1979) Analysis of gene diversity in subdivided populations. Proceedings of the National Academy of Sciences USA. 70:33213323.

Nuez F., R. Morales, J. J. Ruiz, P. Fernández de Córdova, E. Valdivieso y F. I. González (1993) Recolección de especies hortícolas en Ecuador. Plant Genetic Resources Newsletter 96:29-33.

Paris H. (1996) Summer squash: history, diversity and distribution. HortTechnology 6:6-13.
Restrepo J. A., A. Franco y C. Vallejo (2008) Caracterización molecular de introducciones colombianas de zapallo Cucurbita moschata. Acta Agronómica 57:9-17.

Sagástegui A. (1973) Manual de las Malezas de la Costa Norperuana. Universidad Nacional de Trujillo. Trujillo-Perú. 480 p.

Sagástegui A., S. Leiva (1993) Flora Invasora de los Cultivos del Perú. Consejo Nacional de Ciencia y Tecnología (CONCYTEC). Trujillo-Perú. $539 \mathrm{p}$

Sanjur O. I., D. R. Piperno, T. C. Andrés and L. Wessel-Beaver (2002) Phylogenetic relationships among domesticated and wild species of cucurbita (Cucurbitaceae) inferred from mitochondrial gene: Implications for crop plant evolution and areas of origin. Proceedings of the National Academy of Sciences USA 99:535540.

SPSS, Statistical Package for the Social Sciences (2009) IBM Corp. IBM SPSS Statistics for Windows, version 19.0. IBM Corp. Armonk, NY. Disponible en: http://www.softonic.com/s/spss-20. (Diciembre 2012).

Solmaz I. and N. Sarı (2009) Characterization of watermelon (Citrullus lanatus) accessions collected from Turkey for morphological traits. Genetic Resources and Crop Evolution 56:173-188.

Soukup J. (1970) Vocabulario de los Nombres Vulgares de la Flora Peruana. Colegio Salesiano. Lima. $381 \mathrm{p}$.

Uluturk Z. I., A. Frary and S. Doganlar (2011) Determination of genetic diversity in watermelon [Citrus lanatus (Thunb.) Matsum \& Nakai] germplasm. Australian Journal of Crop Science 53:1832 1836.

Ulloa Ulloa C., J. Zarucchi and B. León (2004) Diez Años de adiciones a la Flora del Perú: 1993-2003. Arnaldoa, Ed. Especial 7-242.

Valega Rosas R., T. Andres and M. Nee (2004) The Goldman Cucurbit Collecting Expedition in Peru. Disponible en: http://www.cucurbit.org. (Febrero 2013).

Wang H. X. and T. B. Ng (2000) Ginkbilobin a novel antifungal protein from Ginkgo biloba seeds with sequence similarity to embryoabundant protein. Biochemical and Biophysical Research Communications 279:407-411.

Wang H. X. and T. B. Ng (2001) Examination of lectins, polysaccharopeptide, polysaccharide, alkaloid, coumarin and trypsin inhibitors for inhibitory activity against human immunodeficiency virus reverse transcriptase and glycohydrolases. Planta Medica 67:669-672.

Weberbauer A. (1945) El Mundo Vegetal de los Andes Peruanos. Ministerio de Agricultura. Lima.

Wessel-Beaver L., J. Sud and H. Cuevas (2004) Morphological traits of possible use as species markers in Cucurbita moschata and C. argryosperma. Cucurbit Genetics Cooperative Report 27:54-56.

Yetişir H., M. Şakar and S. Serçe (2008) Collection and morphological characterization of Lagenaria siceraria germplasm from the Mediterranean region of Turkey. Genetic Resources and Crop Evolution 55:1257-1266 